\title{
Rayleigh-Benard convection flow with liquid/solid phase transition in a low gravity field
}

\author{
D. Mansutti ${ }^{1}$, E. Bucchignani ${ }^{2}$ \& M. M. Cerimele ${ }^{1}$ \\ ${ }^{1}$ Istituto per le Applicazioni del Calcolo "M. Picone"/C.N.R., \\ Rome, Italy \\ ${ }^{2}$ Centro Italiano Ricerche Aerospaziali, Capua (CE), Italy
}

\begin{abstract}
The presence of an icy crust covering several satellites of the solar system (e.g. Europa, the jovian satellite) suggested us to accomplish the numerical simulation of the Rayleigh-Benard convection flow of a horizontal layer of mushy water, covered at the top by its own ice and immersed in a low gravity field. The phase transition occurring at the ice/mushy water interface, with release/absorption of latent heat, is included in our study. Starting the simulation with ice and water at rest in a conductive temperature distribution, we pushed the computation forward enough to approach, possibly, the thermo-dynamical equilibrium. We adopt initial geometrical parameters resembling the present set-up of Europa's crust. As the changes undergoing on the satellite nowadays are imperceptible, the final computed configuration should recover the present dynamical and thermodynamical fields. We also provide the estimate of the amount of heat flowing up from the bottom of the domain that is from the inside of the planet, a quantity practically difficult to be measured even on Earth.
\end{abstract}

Keywords: continuum mechanics, phase transition, heat transfer, moving boundary, numerical simulation, natural convection, low gravity, front-fixing, finite differences.

\section{Introduction}

Due to the relevance to techniques in artificial crystal growth and metal manufacturing, in the last three decades specialists in CFD have devoted much 
attention and effort in developing numerical models and computational codes for the simulation of horizontal convection flows with liquid/solid (L/S) phase transition and a bunch of papers on this topic are available in the literature (see, for example [1-4]). Several issues have been deeply investigated, such as the impact of the convection flow of the liquid and of the deformations of the solid on the phase-change process and the dependence of the dynamical and thermal fields on gravity and on the physical and geometrical parameters.

The problem faced in this work, the Rayleigh-Benard convection flow with $\mathrm{L} / \mathrm{S}$ phase change, is linked, as well, to interests of other scientific disciplines, in particular we mention planetology with its queries on the icy satellites of the solar system and geology and glaciology with the studies on the appearance and evolution of the Antarctic subglacial lakes. In both cases occurs the presence of a thick layer of ice with its water underneath, and either the ice or the water undergo a natural heating effect from below that induces vertical convection within both phases. For these applications, the description of the evolution of the icy crust (thickness and deformations), of the convection flow of the water and of heat transfer are, clearly, fundamental. Here, we propose the numerical simulation of the Rayleigh-Benard convection flow of a rectangular laterally open horizontal layer of mushy (freezing) water, covered at the top by its own ice, immersed in a low gravity environment, alike that one of Europa, the jovian satellite. In fig. 1 the geometrical setting is sketched (the phase boundary is drawn as a straight line in its initial shape; with time evolving, it is allowed to become curvilinear).

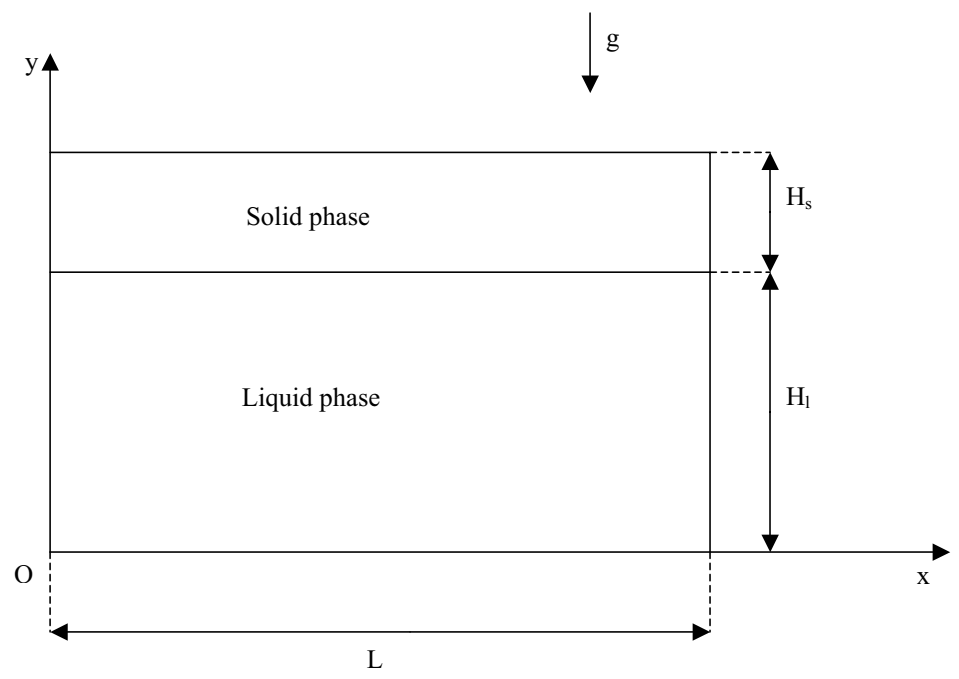

Figure 1: Initial geometrical setting.

In the next section the mathematical equations adopted and the numerical technique used are outlined and, in section 3, the numerical results are presented and discussed. 


\section{Mathematical and numerical modelling}

\subsection{Governing equations}

The mathematical model governing the evolution of a continuum sample undergoing $\mathrm{L} / \mathrm{S}$ phase transition has to include the equations for the conservation laws of momentum, of energy and of mass; for their structure we refer to the books on classical mechanics (e.g. [5]). So a set of equations for each single phase is obtained; they are coupled through the jump conditions, one for each balance equation. In the jump condition for the energy conservation law (so called Stefan condition [6]), most important is the contribution due to release or adsorption of latent heat corresponding respectively to the solidification or the melting processes.

In the model here adopted the liquid phase and the solid phase are respectively described as an incompressible viscous fluid and an isotropic linearly elastic material [5]. This choice allows one to keep average the level of difficulty of the final system of equations to be solved. Obviously, for the ice, a more appropriate model would be one describing correctly the specific material symmetry but, our aim, here, is to provide a first insight to the effects of the mechanical response of the solid phase within the transition process.

Additional simplifying assumptions are:

i) the density of the liquid phase and of the solid phase are assumed to be equal,

ii) the radiating heat is neglected,

iii) the material coefficients of the two phases are assumed to be constant.

Let $t$ and $(x, y)$ be, respectively, the time and the space Cartesian coordinates within a spatial reference frame with origin at the left lower corner of the sample and upward vertical axis. Let us call $D_{L}, D_{S}$ and $\Gamma$ the domains occupied respectively by the melt, the solid and the phase interface. Introducing the Boussinesq and Fourier approximations, the governing equations of the melt flow, holding in $D_{L}$ result:

$$
\begin{gathered}
\rho_{L} \frac{d}{d t} \vec{v}=-\nabla p+\mu_{L} \nabla^{2} \vec{v}-\rho_{L}\left[1-\alpha_{L}\left(T_{L}-T_{p}\right)\right] \vec{g} \\
\nabla \cdot \vec{v}=0 \\
\rho_{L} c_{L} \frac{d T_{L}}{d t}=k_{L} \nabla^{2} T_{L}+\mu_{L}\left[\left(\frac{\partial u}{\partial x}\right)^{2}+\frac{1}{2}\left(\frac{\partial u}{\partial y}+\frac{\partial v}{\partial x}\right)^{2}+\left(\frac{\partial v}{\partial y}\right)^{2}\right]
\end{gathered}
$$

$p, T_{L}$ and $\rho_{L}$ being $\vec{v}=(\mathrm{u}, \mathrm{v})$, respectively velocity, pressure, temperature and density of the melt.

These equations are coupled with the following ones for the solid phase holding in $D_{S}$ :

$$
\rho_{S} \frac{\partial^{2} \vec{U}}{\partial t^{2}}=\mu_{S} \nabla^{2} \vec{U}-\rho_{S}\left[1-\alpha_{S}\left(T_{S}-T_{p}\right)\right] \vec{g}
$$




$$
\begin{gathered}
\nabla \cdot \vec{U}=0 \\
\rho_{S} c_{S} \frac{\partial T_{S}}{\partial t}=k_{S} \nabla^{2} T_{S}
\end{gathered}
$$

$\vec{U}, T_{S}$ and $\rho_{\mathrm{s}}$ respectively being the displacement, the temperature and the density of the solid phase. The symbols $c, k$ and $\alpha$ with the appropriate subscript ( $\mathrm{L}$ or $\mathrm{S}$ ) indicate respectively the specific heat, the conductivity and the thermal expansion coefficients for the liquid and the solid phases, whereas $\mu_{L}$ and $\mu_{s}$ are the viscosity coefficient of the melt and the second Lamé constant of the solid.

The jump conditions, that hold in $\Gamma(\mathrm{t})$, appear:

$$
\begin{gathered}
\vec{v}_{L}=\vec{v}_{S} \\
\left(-p \vec{I}+\mu_{L}\left(\nabla \vec{v}_{L}+\left(\nabla \vec{v}_{L}\right)^{T}\right)\right) \cdot \hat{n}=\mu_{S}\left(\nabla \vec{U}+(\nabla \vec{U})^{T}\right) \cdot \hat{n} \\
-\rho \Lambda\left(v_{L} \cdot \hat{n}-u_{n}\right)-k_{S} \nabla T_{S} \cdot \hat{n}+k_{L} \nabla T_{L} \cdot \hat{n}=0
\end{gathered}
$$

$\hat{n}$ being the normal unitary vector on $\Gamma(\mathrm{t})$ and $\Lambda$ the latent heat. The set of equations (1) -(9) is completed by initial and boundary conditions according to the known requirements of the classical fluid and solid mechanics models (see [5] and further details in the next section).

Let us observe that equation (9) is the well-known Stefan condition; furthermore, let us focus on the fact that, at the phase front, the classical no-slip condition, holding for viscous fluids, within our model implies that interfacing fluid and solid particles are allowed to move sticking to each other with the same velocity. On the contrary in models for L/S phase change that neglect the solid deformations the no-slip condition implies that at the phase front the fluid particles have null relative velocity.

The Coriolis force is not included in the model; this choice is compatible with the application to the icy satellites as it has been shown by Cerimele et al. in [7].

By observing that the vectors $\vec{v}$ and $\vec{U}$ are both required to be solenoidal, we have reformulated the above model on the basis of the Helmoltz-Hodge decomposition in order to meet more accurately and easily such constrain.

This procedure is well known and experimented in fluid dynamics and leads to the scalar potential/streamfunction/vorticity formulation $(\phi, \psi, \omega)$ [8]. In [9] we have proposed to extend this approach also to the treatment of the solid phase by introducing the new unknowns, $\phi_{S}, \psi_{S}$ and $\omega_{S}$ linked to $\vec{U}$ by these relations:

$$
\vec{U}=\left(-\frac{\partial \psi_{S}}{\partial y}+\frac{\partial \phi_{S}}{\partial x}, \frac{\partial \psi_{S}}{\partial x}+\frac{\partial \phi_{S}}{\partial y}\right) \quad \omega_{S}=\frac{\partial U_{y}}{\partial x}-\frac{\partial U_{x}}{\partial y} .
$$

According to this, we transform the equation (4) by applying the curl operator and obtain a scalar equation for $\omega_{S}$. In doing so, equation (5) reduces to a simple Laplace equation for $\phi_{S}$ and $\psi_{S}$ results solution of the Poisson equation coming from the definition of $\omega_{S}$. 


\subsection{Numerical method}

Prior discretization, the mathematical model adopted has been written in nondimensional form by using the following reference quantities: $H_{L}$,

$H_{L}^{2} \rho_{\mathrm{L}} \mathrm{c}_{\mathrm{L}} / \mathrm{k}_{\mathrm{L}}$ and $\mathrm{T}_{0}-T_{m}$ respectively for length, time and temperature.

Then a finite difference method has been applied, with the first order Euler scheme for first order time derivatives, the second order backward scheme for second order time derivatives and centred second order schemes for space derivatives.

Doing so, the unknowns $\omega_{L}, \omega_{S}, T_{L}$ and, $T_{S}$ result explicitly determined from the evolutive equations within a time marching algorithm. The Laplace equations for the scalar potentials and the Poisson equations for the stream-functions, one of each in both phases, are solved by a Bi-CGStab method [10], as linear system solver, supported by a pre-conditioner based on the ILU factorization [11].

In fact the first step in our solving procedure consists in the application of a front-fixing technique that overcomes the difficulties related to the moving boundary formulation: a coordinate transformation is adopted in order to transform the spatial domains, $D_{L}$ and $D_{S}$, into the unitary square and make straightforward the finite difference discretization. Obviously, this transformation generates new factors to the differential operators of the model, which depend on the function of the unknown curve of the phase front. At each time step the discrete points of this curve are properly advanced according to the value of $u_{n}$ obtained by the Stefan condition.

\section{Numerical simulation test}

\subsection{The physical set-up}

The simulation that we have developed is compatible, with respect to either the geometrical and the physical characteristics, with the evolution of a portion of Europa's icy crust, that is two adjacent infinite layers of ice, the conducting one laying above the convective (mushy) one.

Below and above the icy crust are supposed to be respectively the ocean and the atmosphere, not directly included in the simulation; at both boundaries freeslip and impermeability conditions are imposed together with the melting temperature $T_{m}$, at the ice/ocean interface, and the external temperature $T_{w}$, at the top.

At the phase interface, the temperature $T_{\text {top }}$, limiting the convecting ice, is imposed. At the left and right lateral open boundaries, where the infinite layer is artificially truncated, periodicity to $I^{s t}$ order is imposed to the dynamical variables and to temperature. The remaining boundary value of the dynamical variables selects the solution with entire convecting cells.

The values of the geometrical, physical and material parameters, here, follow: $H_{S}=5 \mathrm{Km}$ and $H_{L}=20 \mathrm{Km}$ (initial thickness of ice and mush layers), $L=100$ $\mathrm{Km}$ (length of the truncated layers), $T_{w}=95 \mathrm{~K}, T_{\text {top }}=249.24 \mathrm{~K}, T_{m}=270.5 \mathrm{~K}$, 
$\rho_{\mathrm{L}}=1000 \mathrm{Kg} / \mathrm{m}^{3}, \rho_{\mathrm{s}}=917 \mathrm{Kg} / \mathrm{m}^{3}, \mathrm{k}_{\mathrm{L}}=3.9 \mathrm{~W} / \mathrm{m} \mathrm{K}, \mathrm{k}_{\mathrm{S}}=\mathrm{k}_{\mathrm{L}}, \mathrm{c}_{\mathrm{L}}=4.2103$ $\mathrm{J} / \mathrm{Kg} \mathrm{K}, \mathrm{c}_{\mathrm{S}}=1925 \mathrm{~J} / \mathrm{Kg} \mathrm{K}, \mu_{\mathrm{L}}=10^{14} \mathrm{Kg} / \mathrm{m} \mathrm{s}, \mu_{\mathrm{S}}=10 \mathrm{GPa}, \alpha_{\mathrm{L}}=1.610^{-4} \mathrm{~K}$, $\alpha_{\mathrm{L}}=\alpha_{\mathrm{s}}, \Lambda=3.3410^{5} \mathrm{~J} / \mathrm{Kg}, \mathrm{g}=1.3 \mathrm{~m} / \mathrm{s}^{2}$.

\subsection{Results}

In order to cope with the extremely long computing time required to accomplish the simulation, quite typical of any L/S phase transition computation, we have kept as low as possible the number of discrete unknowns and used time steps as large as possible. The space discretization grid used is $(71 \mathrm{X} 41)$ either in $D_{L}$ and in $D_{S}$; this choice is supported by the grid refinement analysis developed by two of the authors in [7] for a similar problem where the dynamical response of the conductive ice was neglected (eqs. (4-5) and (7-8) dropped). The larger nondimensional time step allowed by numerical stability constrain has been $\Delta \mathrm{t}=$ $\sim 10^{-2}$ (equivalent to $0.25 \mathrm{yr}$ ).

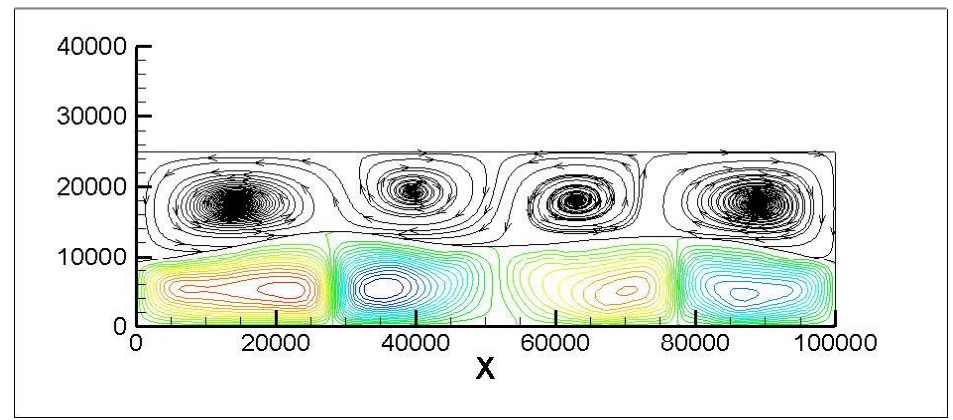

Figure 2: $\quad$ Particle track lines in the solid phase (above) and streamlines in the liquid phase (below) at $\mathrm{t}=17 \times 10^{6} \mathrm{yr}$.

We have started the simulation with conducting temperature profile and both phases at rest and pushed the computation up to time $\mathrm{t}=17 \times 10^{6} \mathrm{yr}$, when symmetry establishes. In fig. 2 we show the tangent lines to the deformation field of the solid phase (particle track lines) and the streamlines of the liquid phase. In fig. 3, the isothermal regions in the whole domain are displayed. We observe the formation of four cellular structures either in the liquid or in the solid phase. Although the elastic response of the solid to the tractive effort operated by the convecting liquid phase at the interface is clearly evident, it is not so important to influence sensibly heat transfer; actually, the thermal field within the solid phase exhibits a conducting behaviour. In the liquid phase two thermal plumes form. As far as that may be deduced by the plots of the thermal field, there is qualitative agreement with the results obtained by Mitri and Showman [12], based on a geology-purpose power-law/finite element code. By looking at the evolution of the phase front, we observe that, approaching the shot time, it still undergoes short oscillations that should be due to the linear elastic nature 
imposed to the ice. So thermo-dynamical equilibrium, if there exists, is not reached yet.

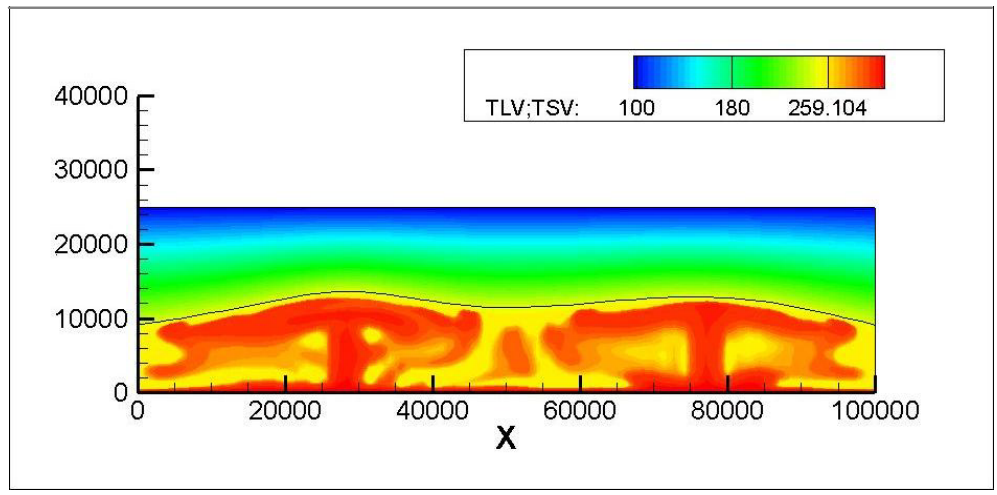

Figure 3: Isothermal regions at $\mathrm{t}=17 \times 10^{6} \mathrm{yr}$.
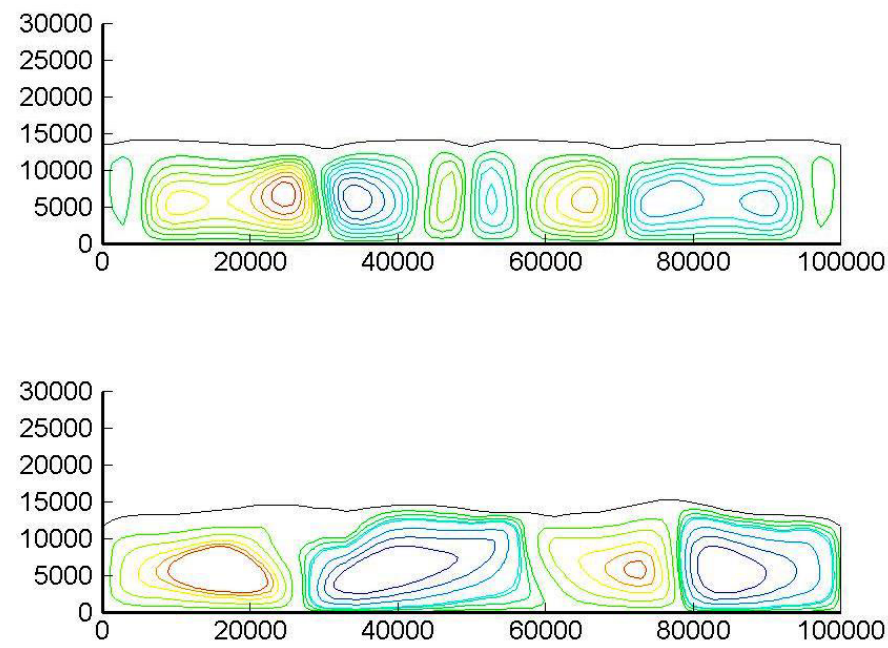

Figure 4: Streamlines in the liquid phase at $\mathrm{t}=2 \times 10^{6} \mathrm{yr}$ (above: solid mechanics not included; below: present model).

In fig. 4 it is possible to compare the streamlines obtained with the present model versus those computed by dropping the solid phase mechanical response: it is evident that this contribution is non-negligible (see the effect on the velocity of phase change) and has to be included in order to simulate correctly the phenomenon considered. 
In fig. 5 the curve of the value of the heat flux, computed at the bottom of the liquid domain, is shown. Its shape is due to the gradients of the thermal field within the lower internal region and, in order to have an estimate of the heat flux coming from the inside of the satellite, it would be necessary to compute the average of the displayed values. We can say, however, that there is just a good match with the range of values extrapolated from the magnetic field data gathered by the Galileo and Voyager spacecrafts, that amounts to (0.02-0.07) $\mathrm{W} / \mathrm{m}$.

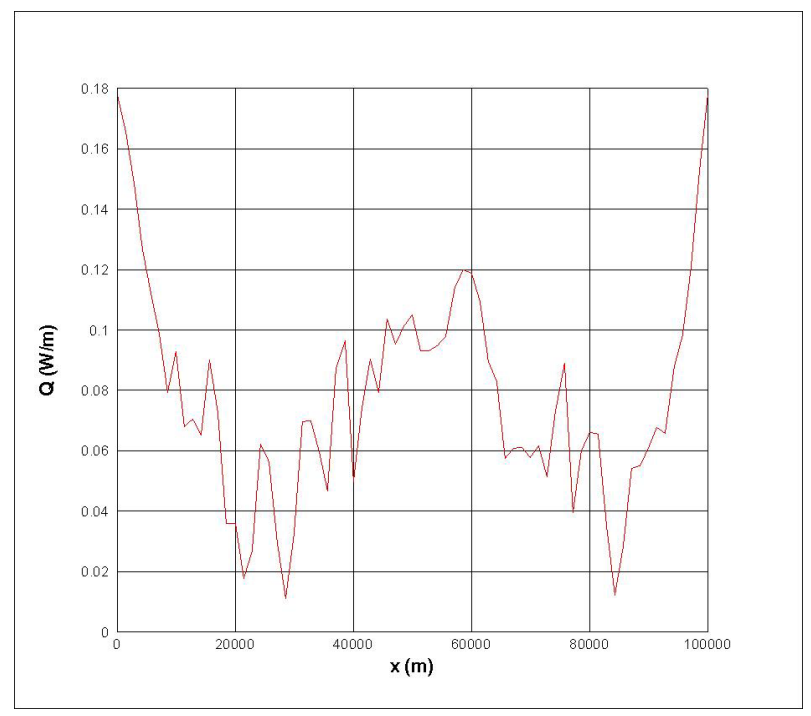

Figure 5: Estimate of the heat flux from the bottom of the domain.

We are aware that a mesh refinement analysis on the present model, at this time under development, is mandatory in order to increase the confidence in our numerical results.

The average CPU time required for running each time step was estimated to be about $0,4 \mathrm{sec}$.

The analysis of the transient regime and the dependence on the classical fluid dynamics non-dimensional parameters ( $\mathrm{Gr}, \operatorname{Pr}$ and $\mathrm{Re}$ ) will be our next challenges.

\section{Acknowledgement}

We gratefully acknowledge Dr. Giuseppe Mitri at the Jet Propulsion Laboratory, Technology Institute of California, Pasadena, for introducing us to the world of the icy satellites of the solar system. 


\section{References}

[1] Gau, C. and Viskanta, R., Melting and solidification of a pure metal on a vertical wall, Transaction of the ASME, 108, pp. 174-181, 1986.

[2] Bertrand, O., Binet, B., Combeau, H., Couturier, S., Delannoy, Y., Gobin, D., Lacroix, M., Le Quere, P., Medale, M., Mercinger, J., Sadat, H. and Vieira, G., Melting driven by natural convection. A comparison exercise: first results, Int. J. Therm. Sci, 38, pp.5-26, 1999.

[3] Kumar, V., Durst, F. and Ray, S., Modelling moving-boundary problems of solidification and melting adopting an arbitrary lagrangian-eulerian approach, Numerical Heat Transfer, Part B, 49, pp. 299-331, 2006.

[4] Mansutti, D. and Bucchignani, E., On the importance of solid deformations in convection-dominated liquid/solid phase change of pure materials, $I A C$ Reports n. 118(12/2006), 2006

[5] Slattery, J. C., Momentum, energy and mass transfer in continua, McGrawHill: New York, 1972.

[6] Crank, J., Free and moving boundary problems, Oxford Science Publication: Oxford, 1984.

[7] Cerimele, M. M., Mansutti, D. and Pistella, F., Study of Europa's crust via a Stefan model with convection, Mathematics and Computers in Simulation, doi:10.1016/j.matcom.2007.11.011, Elsevier Science, The Nederlands, 2007.

[8] Hirasaki, G. J. and Hellums, J. D., Boundary conditions on the vector and scalar potentials in viscous three-dimensional hydrodynamics, Quarterly of Applied Mathematics, 28(2), pp. 163-178, 1970.

[9] Mansutti, D., Raffo, R. and Santi, R., Liquid/solid phase change with convection and deformations: 2D case, Progress in Industrial Mathematics at E.C.M.I. 2004, eds. A. Di Bucchianico, R.M.M. Mattheij, M.A. Peletier, Mathematics in Industry, 8, pp. 268-272, Springer-Verlag, 2006.

[10] Van der Vorst, H., Bi-CGStab: a fast and smoothly converging variant of the Bi-CG for the solution of non-symmetric linear systems, SIAM Jour., 13(2), pp. 631-644, 1992.

[11] Golub, G. and van Loan, C., Matrix computations, The John Hopkins University Press, 1990.

[12] Mitri, G. and Showman, A. P., Convective-conductive transitions and sensitivity of a convective ice shell to perturbations in heat flux and tidalheating rate: implications for Europa, Icarus, 177, pp. 447-460, 2005. 